\title{
Enhancing Sleep Quality for Nursing Home Residents with Dementia: A Pragmatic Randomized Controlled Trial of an Evidence-Based Frontline Huddling Program
}

\author{
A. Lynn Lynn Snow ( $\sim$ LSNOW@ua.edu ) \\ Gordon Palmer Hall https://orcid.org/0000-0002-7383-460X \\ Julia Loup \\ University of Alabama
}

Robert O. Morgan

University of Texas Health Science Center at Houston

Kathy Richards

University of Texas at Austin

Patricia Parmelee

University of Alabama

Rosa Baier

Brown University School of Public Health

Ellen McCreedy

Brown University School of Public Health

\section{Barbara Frank}

B\&F Consulting

\section{Cathie Brady}

B\&F Consulting

Liam Fry

University of Texas at Austin

Megan McCullough

University of Massachusetts Lowell

Christine W. Hartmann

University of Massachusetts Lowell

Study protocol

Keywords: Sleep, Alzheimer's Disease, Dementia, Quality Improvement, Quality Indicators, Health Care, Program Development, Front Line Staff, Nursing Homes, Long Term Care, Mental Health 
Posted Date: October 5th, 2020

DOl: https://doi.org/10.21203/rs.3.rs-68866/v1

License: (c) (i) This work is licensed under a Creative Commons Attribution 4.0 International License. Read Full License 


\section{Abstract \\ Background}

Disturbed sleep places older adults at higher risk for frailty, morbidity, and even mortality. Yet, nursing home routines frequently disturb residents' sleep through use of noise, light, or efforts to reduce incontinence. Nursing home residents with Alzheimer's disease and or related dementias-almost twothirds of long-stay nursing home residents-are likely to be particularly affected by sleep disturbance. Addressing these issues, this study protocol implements an evidence-based intervention to improve sleep: a nursing home frontline staff huddling program known as LOCK. The LOCK program is derived from evidence supporting strengths-based learning, systematic observation, relationship-based teamwork, and efficiency.

\section{Methods}

This study protocol outlines a NIH Stage III, real-world hybrid efficacy-effectiveness pragmatic trial of the LOCK sleep intervention. Over two phases, in a total of 27 non-VA nursing homes from 3 corporations, the study will (1) refine the LOCK program to focus on sleep for residents with dementia, (2) test the impact of the LOCK sleep intervention for nursing home residents with dementia, and (3) evaluate the intervention's sustainability. Phase 1 ( 1 year; $\mathrm{n}=3$ nursing homes; 1 per corporation) will refine the intervention and train-the-trainer protocol and pilot-tests all study methods. Phase 2 (4 years; $n=24$ nursing homes; 8 per corporation) will use the refined intervention to conduct a wedge-design randomized, controlled, clinical trial. Phase 2 results will measure the LOCK sleep intervention's impact on sleep (primary outcome) and on psychotropic medication use, pain and analgesic medication use, and activities of daily living decline (secondary outcomes). Findings will point to inter-facility variation in the program's implementation and sustainability.

\section{Discussion}

This is the first study to our knowledge that applies a dementia sleep intervention to systematically address known barriers to nursing home quality improvement efforts. This innovative study has future potential to address clinical issues beyond sleep (safety, infection control) and expand to other settings (assisted living, inpatient mental health). The study's strong team, careful consideration of design challenges, and resulting rigorous, pragmatic approach will ensure success of this promising intervention for nursing home residents with dementia.

\section{Trial registration:}

NCT04533815, ClinicalTrials.gov, August 20, 2020. 


\section{Contributions To The Literature}

- Tailored sleep interventions combined with systematic data collection and frontline staff huddles can help nursing home staff apply quality improvement techniques to reduce the negative health impacts of disturbed sleep for residents with Alzheimer's disease and related dementias

- This hybrid efficacy-effectiveness pragmatic trial systematically addresses the known barriers to nursing home quality improvement efforts (e.g., staffing problems, exclusively top-down approaches hampering frontline involvement, and being divorced from frontline realities).

- The study design has future potential to be applied to important clinical issues beyond sleep (safety, infection control) and in other settings (assisted living, inpatient mental health).

\section{Background}

A layman's case for the benefits of a good night's sleep is easy to make: imagine someone woke you last night every two hours. How would you feel? Now imagine that routine continues every night for the next month. For many residents of nursing homes, such awakening is standard practice. Nursing homes implement such segmented sleeping routines to address various care challenges (e.g., incontinence), even though evidence from both objective and subjective measures identifies disturbed sleep as a key contributor to many types of physical, emotional, and cognitive decline (1-4), including risk for frailty, morbidity, and even mortality (5-10). Individuals with Alzheimer's disease and related dementias (hereafter referred to as 'dementia') -almost two-thirds of long-stay nursing home residents (11) - are particularly affected by sleep disturbance $(12,13)$. Poor sleep quality in this population is associated with increases in self-reported fatigue, difficulties with activities of daily living (ADLs), depression, fall risk, and, together with decreases in memory, mobility, morbidity, and even rates of survival $(3,14-17)$.

Various factors create suboptimal sleeping conditions for residents in nursing homes, including efforts to reduce incontinence (18), environmental disturbances such as frequent noise and light (19-21), and residents spending much of the day without engaging in physical activity $(22,23)$. Nursing home residents with dementia are more likely to receive antipsychotic or antidepressant medications compared to other residents (24); these medications may worsen nighttime sleep (25), In addition, withdrawal from antipsychotic medication may temporarily worsen sleep (26). Pain also may affect sleep through nighttime disturbance and reduced physical functioning during the day (27). The relationship between sleep quality and physical functioning (e.g., activities of daily living) is also well-established, with poorer objective sleep associated with poorer physical functioning in older adults $(3,6,28,29)$ and those with dementia (30).

Despite the common knowledge that sleep disturbance has negative impacts on nursing home residents, and despite the availability of easy ways to measure (e.g., actigraphy tools) and intervene to increase sleep quality, little has been done to improve the situation. A 2018 systematic review of nonpharmacological sleep interventions in nursing homes indicated promise for system-level environmental changes such as increased daytime light exposure, nighttime use of melatonin, and 
acupressure (31). But another 2018 review noted that sleep disturbance remains extremely common for nursing home residents (32). Even when nursing homes do recognize the need for systems-level changes and act to make those changes, their efforts are often translated into a one-size-fits-all approach to implementation, ultimately overlooking the complex interplay between individual residents' needs, staff availability, and environmental barriers (33-35). Because good sleep results from a complex interplay of resident, staff-generated, and environmental factors (33), sleep regimens for nursing home residents work best when individualized instead of applied generically.

In response, this study aims to improve awareness and measurement of sleep through implementation of a sleep-focused, frontline staff huddling intervention, systematic measurements of individual nursing home residents' sleep, and targeted intervention sustainment through follow up visits (which may include virtual visits) with participating nursing homes.

\section{Huddling and the LOCK Program}

Quality improvement (QI) barriers such as staffing problems and top-down approaches can hamper nursing homes' efforts to enhance care quality $(36,37)$. QI studies in nursing homes have responded to these barriers by underscoring the importance of open communication and relationship building to improve resident clinical outcomes $(36,38,39)$. A recent systematic review highlights key components for successful nursing home Ql: changing staff behavior, targeting specific care tasks, and using intervention theories (40). I One QI practice that incorporates these components, and therefore has potential to meaningfully impact resident sleep, is frontline staff huddling. This type of huddling involves brief, standup meetings that facilitate efficient, collaborative information exchange about specific topics, concerns, or resident needs. Frontline staff huddling promotes communication across clinical roles (42-44) and improvements in clinical outcomes across residents (45-47). More broadly, consistent huddling can help improve quality of care and sustain changes individually and systematically by directly involving frontline health care workers (41).

In a prior series of studies $(48,49)$, we incorporated the evidence-based practices of strengths-based learning $(50,51)$, systematic observation (52), efficiency $(49,53)$, and relationship-based teamwork (54, $55)$ into a specific frontline staff huddling program known as the LOCK program. The LOCK program enables staff to (1) Learn from bright spots (focus on positive evidence of strengths); (2) Observe(collect data through systematic observation); (3) Collaborate in huddles (conduct frontline staff huddles); and (4) Keep it bite-size (limit activities to 5-15 minutes for efficiency) $(49,56)$. (See Figure 1; LOCK Elements, Corresponding Evidence-Based Concepts, and Examples). The LOCK program actively guides frontline staff through a process of addressing particular resident outcomes of concern-in the case of this proposal, sleep-using frontline staff huddling on a daily, weekly, or other applicable consistent schedule as the foundation. Beyond the focused impact on residents' sleep, our use of the LOCK program aims to increase nursing home staff application and use of the LOCK huddling method.

A pilot LOCK program targeting staff-resident interactions in 6 Veterans Health Administration (VA) nursing homes resulted in meaningful quantitative and qualitative improvements (48). The 6 
participating nursing homes showed significant pre- and post-intervention improvements in interpersonal interactions with residents for whom staff were providing care and reductions in the quantity of negative staff interactions with residents. The LOCK program was subsequently rolled out to all 134 VA nursing homes using a train-the-trainer approach. With the support of an intervention team that included national experts in nursing home QI implementation, geriatric sleep, and clinical dementia interventions, the rollout of the LOCK program expanded to focus on a variety of resident clinical conditions such as pain and pressure ulcers. The LOCK program's methods have also improved clinical care in several non-VA nursing homes, including those with a history of serious, intransigent quality issues $(41,57,58)$.

The combined results of the series of preliminary work with the LOCK program (1) enabled our team to standardize the LOCK program, (2) resulted in qualitative data on best practices for LOCK program implementation in nursing homes, and (3) generated implementation materials, all of which we will use for the present study to systematically measure sleep outcomes data in the context of our targeted LOCK sleep program.

\section{Intervention}

This clinical trial is a pragmatic study with two consecutive phases of refining and implementing the LOCK sleep intervention. Phase 1 consists of the following milestones over a period of one year: identifying pilot sites, initiating and completing intervention training, testing primary data collection, and transferring data. Upon successful achievement of these milestones, a four-year Phase 2 will test the impact of implementing the LOCK sleep intervention on nursing home residents' sleep outcomes. Phase 2 will implement a pragmatic trial in 3 multi-facility, community-based, nursing home corporations caring for residents with dementia. In collaboration with those corporations, we will conduct a three-wave wedge-based, cluster randomized, controlled trial (RCT) with 24 nursing homes.

Our design enables testing of the extent to which the LOCK sleep intervention, after refinement for the nursing home resident dementia population, accomplishes the following:

1. improves sleep quality as assessed by actigrapy (primary outcome);

2. reduces psychotropic, hypnotic, and analgesic medication use (secondary outcome); and

3. minimizes residents' pain, mood, behavior symptoms, skin breakdown, and decline in activities of daily living (ADL) (secondary outcomes).

Results will additionally provide insight into variation of the intervention's implementation and sustainability across care settings, nursing home organizational structure, and resident cases. Measurement of the implementation success of our LOCK sleep intervention will be guided by the Consolidated Framework for Implementation Research (CFIR) (59-61).

\section{Intervention Strategies}


The LOCK sleep intervention incorporates strategies known to be effective in healthcare organizations and for behavioral change at both a staff and resident level. To further support the its successful implementation, we built several strategies into the study design to efficiently target nursing home staff interaction, improve sleep outcomes for nursing home residents with dementia, and support sustainment: (1) frontline staff involvement, (2) staff training, (3) video dissemination, (4) novel technology testing, and (5) sustainment.

\section{Frontline staff involvement}

The LOCK sleep intervention explicitly supports frontline staff involvement through a structured frontlineinclusive staff huddling process that ensures collaborative and resident-individualized problem solving. The huddles will be regular short stand-up meetings occurring a few times each week and will include the medical interdisciplinary team, front-line staff, and any other staff who have resident relationships and knowledge (e.g., activities, custodial). Such direct staff involvement is central to achieving improvements in quality of care and naturally integrates open communication and connectivity into care teams' daily dialogues $(38,39)$. Inclusion at the frontline level allows for important information about resident's needs, preferences, and habits to be incorporated in solutions, while also avoiding the more commonly utilized, and less successful, exclusively top-down QI strategies (36).

\section{Staff training}

During study Phase 1, we will train corporate coaches from each nursing home corporation on the LOCK sleep intervention. Coaches will be local nursing home employees primarily responsible for the provision of training and regular supervision and support for all site leadership teams. They will also provide oversight of the program at all sites during Phase 2, with the support and weekly supervision of our intervention consultants. Coaches will guide the leadership in each nursing home site by creating a study leadership/implementation team, training and mentoring those teams, and working with those teams to track data and reviewing progress to make midcourse corrections (47). This train-the-trainer approach requires only minimal facilitation from the research team and consultants, thereby mimicking real-world conditions and enhancing the potential for future sustainment and dissemination of the intervention.

\section{Video dissemination}

We will create 10-minute videos of nursing homes' successful use of the LOCK sleep intervention over the course of Phase 1 and 2. The videos will be used during the study as education for fellow participating nursing homes and disseminated nationally after study completion.

\section{Novel technology testing}

After piloting of the LOCK sleep intervention, exploratory qualitative interviews with staff will investigate nursing home impressions of Fitbits in comparison to actigraphy. Fitbits (Fitbit Inc., San Francisco, CA) are an example of the large class of affordable and widely available consumer-level accelerometers, as contrasted with the class of medical-level accelerometers typically used by sleep laboratories (e.g., to 
collect our primary outcome sleep measurements we will use the Micro-Mini Motionlogger Actigraph by Ambulatory Monitoring Inc., Ardsley, NY). To our knowledge, no studies have examined nursing home staff-perceived benefits of such devices. Although anthropometry studies have demonstrated Fitbits to be inferior measurement devices for precise sleep research measurement purposes (62-64), they might be useful for real-world clinical intervention implementation because they are more financially accessible for nursing homes and allow staff to more easily and understandably interact with the residents' sleep data in meaningful ways (e.g., user friendliness of design, multiple types of data, data visualization) (65).

\section{Sustainment}

We plan to investigate the LOCK sleep intervention's sustainment in each nursing home six months after completion. Sustainment of an intervention-the extent to which it becomes part of usual care-is a little studied but significant area of implementation research (66-68). During this follow up, we will conduct semi-structured interviews with participating nursing home staff to elucidate barriers to and facilitators of the intervention's sustainment (or lack thereof).

\section{Methods}

\section{Setting of the LOCK Intervention}

The LOCK sleep intervention will be implemented in a total of 27 nursing homes. We will recruit 3 nursing home corporations that have (1) a minimum of 12 nursing home sites ( 8 will eventually participate in the study), (2) $50+$ beds, (3) $\geq 30$ long-stay residents with dementia diagnoses, (4) centralized corporate training staff that can devote $50 \%$ time to implement the intervention (corporate coaches). Once the corporations are confirmed, eligible nursing homes within the corporations will be identified for recruitment of Phase 1 and 2. We will finalize nursing home eligibility criteria with the selected corporations using the nursing home's Minimum Data Set (MDS) and Certification and Survey Provider Enhanced Reporting (CASPER) self-reported data as the trial progresses (see Data Collection for further details). Leaders at each participating site (i.e., administrator, chief nurse, medical director, nurse managers) will commit to (a) researcher-determined scheduling of intervention participation as per the randomization schedule, (b) taking on identity and responsibility of site leadership team, and (c) work with the study staff to execute secure data transfer/shipping protocols. Working with corporations provides the advantage of shared corporate infrastructure across nursing homes to facilitate screening, implementation, and data collection.

Nursing home recruitment for Phase 1 will require cooperation from the 3 enlisted corporations to identify 3 individual nursing homes (1 per corporation) to participate in the piloting and refining of the LOCK sleep intervention. Once identified, the study intervention team (study co-investigators and consultants with clinical and implementation expertise with the LOCK sleep intervention) will lead the Phase 1 implementation of the intervention. Stakeholder engagement will occur at both corporate and the nursing home levels during this phase. At the corporate level, each corporation will assign a corporate coach to attend all nursing home visits (which may be virtual visits) with the implementation team at their 
corporation's selected Phase 1 nursing home. This allows training of corporate coaches to ensure preparedness and organizational support for the nursing home when they enter study Phase 2 . At the nursing home level, each nursing home will create site leadership and implementation teams to support involvement of all staff in the LOCK sleep intervention.

Nursing home recruitment for Phase 2 includes expanding our sample to an additional 24 nursing homes, 8 from each corporation, using the same inclusion criteria as Phase 1. Once selected, each nursing home will receive the piloted and refined LOCK sleep intervention.

\section{Resident Sample}

In Phase 1, we anticipate enrolling 57 residents with dementia who meet inclusion/exclusion criteria (approximately 19 per each of 3 nursing homes). In Phase 2, we anticipate enrolling 456 residents with dementia (approximately 19 per each of 24 nursing homes). An approximation of 19 residents per nursing home is calculated according to an averaged $50 \%$ prevalence of dementia within nursing homes and conservative $50 \%$ consent rate based on our study team's experience with previous nursing home dementia trials $(69,70)$. We have included an estimate of approximately $20 \%$ attrition across the two study phases to maintain statistical power (71).

The potential participant pool from each selected nursing home will include all residents aged $>=50$ years with a dementia diagnosis. Because the LOCK sleep intervention allows staff to focus on residents with dementia who have the greatest sleep problems, without differentiating by dementia severity, we will include residents across the range of dementia severity. Nursing home staff will be trained to identify nursing home residents with high risk of obstructive sleep apnea using the STOP-Bang screening tool (7274), as well as trained on appropriate procedures for referring any positively screened residents for medical evaluation. We will exclude residents with a high risk of obstructive sleep apnea who are not yet being treated for said condition due to evidence of inaccurate actigraph measurements in that population (75). Staff will also exclude residents who have a persistent bilateral resting tremor or paralysis in both arms (a subset of persons with Parkinson's disease and related significant tremor-causing diagnoses), due to similar actigraph measurement inaccuracies (76).

Due to inclusion of residents with dementia diagnoses, a two-step resident consent process will be used to address the ethical balance between beneficence, assuring that residents who do not have the capacity to consent are protected by their legal authorized representative's oversight, and promotion of autonomy for residents who do have capacity to consent. First, after leadership teams from each nursing home compiles a list of all residents with a dementia diagnosis, they will send a letter to each resident's legally authorized representative informing them of the study and inviting the legally authorized representative to opt out if they do not wish to be contacted by research staff (77-80). Research staff will then contact all representatives who do not opt out, inviting each to consent to the resident's participation. Once legally authorized representative consent is collected, the second consent stage for nursing home residents with mild dementia will occur. Site leadership team members will work with our study team to identify those residents with a mild MDS Brief Interview for Mental Status (BIMS) score (i.e., a score above 10) and a 
resulting increased likelihood of capacity to consent (81). Nursing home leadership teams will then arrange a telephone interview between study staff and these residents to ascertain the resident's ability to consent using comprehension questions. If the resident is determined to have the ability to consent during this interview, the resident consent process formally commences at that time, with a nursing home staff person serving as in-person witness.

Importantly, if a resident can respond appropriately to all capacity screening questions but does not wish to participate, they will not be enrolled in the study, regardless of their legally authorized representative's consent. For nursing home residents without capacity to consent that become enrolled in the study, their assent to participate will be monitored during the study at each step, and their decision to withdraw at any time (whether expressed verbally or by resistance to participation) will be honored.

\section{Staff Sample}

We will enroll 60 frontline staff in the Phase 1, piloting and refinement year, of our clinical trial. In Phase 2, we will enroll 480 staff in our clinical trial across the 24 nursing homes. We will invite all frontline $\mathrm{NH}$ staff roles interested in the training and intervention to enroll (e.g., Nursing Assistants, Licensed Practical Nurses, Registered Nurses, and other interdisciplinary frontline staff members). We will recruit 20 staff per nursing home to participate in study interviews about their experiences with the LOCK sleep intervention and Fitbits at mid- and at six-months post-implementation.

To increase enrollment and retention of staff, we will communicate the compelling, evidence-based benefits of the LOCK sleep intervention on improved work environments and quality of care for nursing home residents. The regular phone mentoring of nursing home corporate coaches and leadership teams will also facilitate staff retention and intervention fidelity. Consent is not required from staff members directly participating in the LOCK sleep intervention as the program itself consists of clinical practices common in nursing home QI efforts that fall within the scope of staff duties. However, it will be made clear that participation is strictly voluntary and no adverse consequences to the person's job status or any other adverse consequences will occur if the person declines to participate in the LOCK sleep intervention at their nursing home facility. At the end of Phase 1 and Phase 2, research staff will obtain consent from the sub-sample of nursing home staff participating in interviews.

\section{Measures, Data Collection, and Analysis Methodology}

\section{Study Design.}

The LOCK sleep intervention. In Phase 1, the LOCK sleep intervention will initially use intervention materials (assistance for developing teams and implementing the intervention, checklists, role-play scenarios, and videos) developed from the pilot 6-site VA nursing home implementation [34]. Throughout this phase, we will make additional refinements based on our intervention team's experiences from their previous work. We will also carefully adapt our materials to the non-VA nursing home setting, which 
differs in terms of resident population (VA is mostly male), staffing (VA has higher professional nurse staffing ratios), and leadership (VA medical director is usually on site).

Phase 2 will test the refined LOCK sleep intervention through an incomplete, stepped, wedge cluster randomized controlled trial (RCT) design (82) with 24 additional nursing homes, each acting as its own control (6 staggered steps, with 4 nursing homes per step) (See Table 1: Stepped Wedge Design with Measurement Periods). During Phase 2, each cluster of 4 nursing homes will participate in its own 22week intervention cycle (see Figure 2: Timing and Spacing of Intervention Implementation and Sleep Outcomes Measures) after being assigned first to the control condition and then phased into the intervention at 7-week intervals. We will randomize nursing homes within corporations to steps after matching on bed size and number of long-stay residents with dementia diagnoses. Residents within each nursing home are followed longitudinally, i.e., from pre- through post-intervention measurement periods.

Outcome Measures. The primary outcome for the study is actigraph-measured resident sleep. Total sleep time will be measured using data from Micro-Mini Motionlogger Actigraphs worn by residents at night (Ambulatory Monitoring Inc., Ardsley, NY) (83). We will define nighttime as $10 \mathrm{pm}$ to $6 \mathrm{am}$ and will compute total sleep time as the total number of minutes asleep during a nighttime period. To further evaluate the impact of the LOCK sleep intervention for resident sleep quality, we will also examine the following: wake after sleep onset (total number of minutes awake during nighttime), how often the resident awoke during the nighttime, sleep efficiency (the ratio of minutes asleep to minutes awake over the period), and sleep fragmentation (an index of restlessness computed as the percentage of one-minute epochs scored as awake).

Due to imperfect correlations between actigraphic sleep data and self- or other ratings of resident sleep $(84,85)$, four supplementary sleep measurements will be collected from staff to triangulate the primary sleep outcome data: (a) Sleep via staff rating: a clinical, global impression of resident sleep change at the end of each 7-night actigraph sleep measurement period and at the end of each week of the 6-week sleep intervention period (86-93); (b) Staff-identified sleep-related concerns: Huddle facilitators lead the huddle team to identify and track up to 2 additional symptoms or behaviors of concern potentially related to sleep (e.g., nighttime agitation, pain), measured on the same schedule as the 'sleep via staff rating'; (c) Inter-resident sleep variability: Medical record data to be collected at the end of the 22-week trial measurement period on the following: (1) changes in any sedating medications and/or dosages; (2) incidents of delirium; (3) any urinary tract infections; and (4) doses of any sedating medications, including as needed ones; (d) Sleep from the Minimum Data Set (MDS): Two MDS items pertaining to sleep will be included: (1) "trouble falling or staying asleep or sleeping too much."; (2) "over the past 5 days, has pain made it hard for you to sleep at night?"

Our secondary outcomes will come from the nursing homes' MDS 3.0. Every Medicare-certified nursing home is required to complete an MDS assessment for every resident at admission, quarterly, and at discharge, in addition to whenever the resident's status changes. We will be using the MDS to collect details about the residents': (1) psychotropic medication use in the last seven days; (2) self-reported pain 
index $(94,95)$; $(3)$ adherence to a regimen or isolated use of analgesic medication in the last five days, noting use of additional non-medication pain interventions; and (4) indication of decline in activities of daily living (ADLs) when considering past MDS assessments and overall functional ability (96-99). (See Table 2: Primary, Secondary, and Other Outcome Measures).

Semi-structured interviews will also provide the qualitative data needed for our team understand the fit and effectiveness of the LOCK sleep intervention during both Phases 1 and 2.

Data Collection. Data collection for the primary sleep outcome measures will follow the same timeline in Phases 1 and 2, incorporating three measurement periods: pre-intervention, intervention, and sustainment. We anticipate an initial 8-week period in which each nursing home builds its frontline staff huddle practice across all units (pre-implementation period). In the first and last week of this period, nursing home staff will conduct a week (7 days) of continuous actigraph measurement on all enrolled residents in each nursing home. These will be the pre-baseline and baseline measurements. After this, each nursing home will begin the LOCK sleep intervention on all its units (implementation period), which involves no actigraph measurement. An 8-week sustainment period will follow. In the first and last weeks of the sustainment period, nursing home staff will also conduct a week (7 days) of continuous actigraph measurement on all enrolled residents in each nursing home. These will be the post-intervention and sustainment measurements. (See Figure 2: Timing and Spacing of Intervention Implementation and Sleep Outcomes Measurement).

Nursing home staff will assist residents to wear both an actigraph and a Fitbit (if applicable during Phase 2) side by side on a wrist for the 7 days during each of the 4 measurement periods (weeks 1, 8, 15, and 22 -see Figure 2) (100-102). As part of their intervention training, staff will be instructed on behavioral signs of distress. If a participant exhibits significant behavioral distress due to sleep measurement methods, staff will be instructed on modifications and alternatives. If the distress does not subside, staff will be instructed to discontinue the assessment. There will therefore be no alternative treatments due to the stepped-wedge, cluster randomized, controlled clinical trial design.

To record collected sleep and secondary data, the nursing home leadership team will use a researcherprovided, tracked, express mail service to send the research team all weekly completed staff rating forms. These mailed packages will also include any actigraphs and Fitbits for which the assessment periods are complete. For supplementary sleep data collection, research staff will additionally extract relevant information from each enrolled resident's medical record.

For qualitative data collection, we will recruit a sample of nursing home staff engaged in huddles to participate in mid- and postimplementation semi-structured interviews (phone or in-person, as situations permit) to explore staff perceptions of the LOCK sleep intervention's effectiveness, feasibility, facilitators, and challenges. During Phase 1, reviews of the Fitbits and suggestions to improve the interview process for Phase 2 (i.e., change in length, location, etc.) will also be collected. 
Data Management. The principal investigator will be responsible for ensuring participants' safety on a daily basis. An independent Data and Safety Monitoring Board (DSMB) will meet at least once a year by teleconference (and more often as needed), acting in an advisory capacity to the NIA Director by (1) monitoring participant safety; (2) evaluating the progress of the trial; and (3) reviewing procedures for maintaining the confidentiality of data and the quality of data collection, management, and analyses. The DSMB meetings will be guided by the NIA's DSMB Report Template, including but not limited to: study status and stopping guidelines, recruitment status, data quality status, interim results, and safety information. This study's DSMB members have been approved by the trials' assigned NIA program officer as independent investigators with no scientific, financial, or other conflict of interest with the study.

Personally identifiable information from the trial will be only be stored in a secure and securely backed up University of Alabama data server and University of Texas Health Data Center in password protected files. Other collaborating sites (Boston University, Brown University, and University of Texas at Austin) will only work with collected data identified by subject number. All data, and referenced personal information therein, will be monitored by the DSMB during and after the trial. HIPAA-compliant virtual visits will assist in streamlined data collection during reduced in-person access to nursing homes due to the COVID-19 pandemic.

Analysis. In Phase 1 we will use standard microlongitudinal analysis methods to test the sensitivity of secondary outcomes and supplementary sleep data to individual differences and changes in residents' total sleep time. For the qualitative interviews, all interviews will be audio recorded, transcribed, and processed through rapid appraisal template analysis $(103,104)$. The Consolidated Framework for Implementation Research (CFIR) constructs, a widely used theory for evaluating intervention implementation $(71,105)$, and the Relational Coordination framework will form the foundations of the analysis template $(106,107)$ (See Figure 3: Relational Coordination Theory). Results from this step will suggest if the value and feasibility of using the Fitbits in addition to the actigraphs supports their continued use in Phase 2.

After Phase 2 data collection is complete from all 24 nursing homes, we will examine differences in the primary outcome (total sleep time) across the pre-baseline through each nursing homes sustainment period (See Figure 2). We will use the nursing home's separate average total sleep times across each of the four measurements. We will use a multi-level mixed models approach and either generalized linear mixed models (GLMM) or generalized estimating equations (GEE). Both GLMM and GEE approaches enable the estimation of models with random and fixed effects and the use of outcome measures having the range of distribution types likely to be observed in primary and secondary outcomes (105).

The level of analysis will be the individual nursing home resident nested within nursing home corporations, with each nursing home serving as its own control and nursing homes stratified by corporation. Data available from the pre-baseline, baseline, postintervention, and sustainment measurements (See Figure 2 and Table 1) will enable estimation of (a) within individual variation over time (pre-baseline [week 1] through sustainment [week 22]), (b) between individual variation incorporating 
individual characteristics (e.g., demographics, dementia severity level), and (c) facility level variation incorporating nursing home characteristics (e.g., corporate membership, facility bed-size, and staffing) $(108,109)$.

Since using actigraphs to measure sleep time will likely be a new practice in the nursing homes, the two pre-intervention measurement periods (pre-baseline [week 1] and baseline [week 8]) will enable us to examine the impact of measuring sleep time prior to the intervention separate from the effects of nursing home staff implementing the LOCK sleep intervention. The two post-implementation periods (postintervention [week 15] and sustainment [week 22]) will enable us to test for longer-term intervention effects (sustainability).

Our primary analysis for trial evaluation will be a partial intention-to-treat analysis using total sleep time as the outcome. We will include all residents with both pre-baseline and baseline total sleep time assessments to properly estimate any effect of using the actigraph to measure sleep. To adjust for midtrial attrition, we will use multiple imputation to estimate missing data, assuming no change from their last available total sleep time assessment and incorporating an estimate of random variation based on observed data. We will apply our model approach described above to the multiply-imputed datasets and results will be combined using Rubin's Rule (110). A complete case analysis (individuals with data from all measurement periods) will be conducted to test the sensitivity of our primary analysis results.

Participating nursing homes will be treated as random effects with residents clustered within nursing homes. By clustering within nursing home, we can control for site-level effects and plausibly treat the individual residents as independent (71). We will estimate both unadjusted and adjusted models. We will include individual-level and nursing home-level characteristics in our adjusted models, as described above. A Type I error rate of $5 \%(a<.05)$ will be used to identify statistically significant associations. Raw and adjusted total sleep times will be reported for each period. We will use a similar approach for testing the impact of the LOCK sleep intervention on our secondary outcomes and our supplementary sleep data. For our secondary outcomes from the MDS, we will use data from each resident's last available MDS assessment pre-intervention and the first available MDS post intervention. Additional MDS data may be included to extend the pre- and post-implementation assessment windows, increase our ability to distinguish individual variability from intervention-related change, and provide greater statistical power for these analyses. The form of the models tested will depend on the completeness of MDS data available for each participant.

We will triangulate the quantitative and qualitative data using thematic analysis according to responses associated with variation in the program's implementation and sustainability (103).

Implementation Assessment. For Phase 2, we will assess implementation success using $\operatorname{CFIR}(59,60)$ according to the entire sample of 24 nursing homes. Our analysis will (1) characterize nursing home variation in implementation measures based on selected constructs within 4 CFIR domains (61) and (2) use multiple data sources to document and evaluate the implementation sustainment (Table 3: CFIR Implementation Constructs and Data Sources). In addition, we will use methods outlined by Keith et al. to 
create a measure of variability (111) using both qualitative and quantitative data. This measurement will reflect how the intervention was used in practice, how it was sustained, and identify influences for our results. The analysis methods will apply to both the post-intervention and the sustainment analyses. We will triangulate perspectives on the CFIR constructs, to the extent possible, using the different data sources. For the qualitative data, triangulation requires first using the taxonomy of themes to assign quantitative ratings and averaging them to create a ranking. For the quantitative measures, we will empirically test for correlation and create a rating by weighting the correlated measures and creating a summary ranking.

Summary rankings will enable us to describe the variability in implementation among the trial's nursing homes, both at the overall nursing home level and at the individual CFIR construct level. We will average the rankings across all constructs for each nursing home and then rank all nursing homes based on the average scores. This ranking process, crossed with staff impressions of the Fitbits, and rich qualitative data to provide context, will provide an overall summary of variability across nursing homes (112-114). As a final step, we will examine how this study's implementation metrics align with quantitative results for our primary, secondary, and supplementary sleep data outcomes, helping us understand how the nursing homes implemented the LOCK sleep intervention and how the program was sustained. This will inform expansion of the intervention as well as inform other nursing home QI initiatives.

\section{Discussion}

This trial's rigorous evaluation of the LOCK sleep intervention will establish its effectiveness for improving sleep for nursing home residents with dementia and will characterize factors associated with effective intervention implementation and sustainment. Innovative, real-world studies like this are critical to assist nursing home staff in improving the quality of care for this vulnerable and growing population.

Our design and implementation plan are strengthened by an emphasis on implementation and intervention sustainment. Sustained use of the LOCK intervention will suggest the likelihood of its viable expansion within nursing homes to focus on other health and behavioral issues that nursing home staff and residents face, such as safety and infection control. It will also point to potential applications in settings other than nursing homes, such as assisted living and inpatient mental health units. In terms of other possible positive outcomes, the LOCK intervention's emphasis on improved communication and teamwork may enable improved work experiences and therefore may lead to reduced staff turnover (115). Successful implementation is also likely to benefit residents by facilitating better and earlier detection and treatment of sleep problems, as well as increasing staff's attention to residents' physical and mental health conditions.

Our innovative study also includes the use of a novel technology, the Fitbit, that has yet to be evaluated for use in nursing home sleep trial. Results will determine Fitbits' potential value in terms of both design and access (financially, bodily) for this population and the nursing home setting. 
Regarding study limitations, the emphasis on real-world implementation necessarily means some loosening of intervention implementation fidelity requirements. For example, depending upon the state of COVID-19 protection procedures during the course of the study, research staff may have little to no opportunity to visit participating nursing homes. In a pragmatic design, identification of specific active ingredients for change is not always possible, as is true in our case, where the intervention consists of several practices (sleep hygiene instruction, team huddling procedures, sleep measurement procedures).

\section{Conclusion}

Our interdisciplinary team of researchers and consultants designed this study to improve clinical outcomes for nursing home residents with dementia. This study's central innovation, the LOCK sleep intervention, holds promise for being an effective, real-world mechanism to improve sleep for nursing home residents and significantly move forward goals of the National Plan to Address Alzheimer's Disease 2018 Update (116). Upon implementation completion, this intervention has future potential to address other important issues faced by residents and expand to settings. Our strong research team, careful consideration of design challenges, and rigorous, pragmatic approach will work together to ensure success of this intervention. Efficacious implementation of our evidence-based intervention to improve residents' sleep will significantly increase research understanding of how to implement and sustain nursing home interventions and improve quality of life and sleep for this important, growing, and vulnerable population.

\section{Abbreviations}

$\mathrm{ADL}$

Activities of daily living

AHCA

American Health Care Association

CASPER

Certification and Survey Provider Enhanced Reporting

CFIR

Consolidated Framework for Implementation Research

DSMB

Data Safety Monitoring Board

GEE

Generalized estimating equations

GLMM

Generalized linear mixed models

LOCK

"Learn from bright spots", "Observe", "Collaborate in huddles", and "Keep it bite-size" MDS 
Minimum Data Set

NIA

National Institute on Aging

RCT

Randomized Controlled Trial

\section{Declarations}

\section{Ethics Approval and Consent to Participate}

This study was granted full board approval by the University of Alabama Medical Institutional Review Board; IRB Reference Number: 19-024-ME. Collection, assessment, and managing of solicited and spontaneously reported adverse events, including required protocol alterations, will be communicated and approved directly with the IRB, trial's data safety monitoring board, and NIA funding agency.

\section{Consent for publication}

Not applicable.

\section{Availability of Data and Materials}

The datasets generated and/or analyzed during the current study are not publicly available because the current protocol has yet to begin. They will be available from the corresponding author on reasonable request after trial data collection has completed.

\section{Competing Interests}

The authors declare that they have no competing interests.

\section{Funding}

Research reported in this publication was supported by the Institute on Aging of the National Institutes of Health under Award Number: R61AG065619. The content is solely the responsibility of the authors and does not necessarily represent the official views of the National Institutes of Health.

\section{Adherence to Reporting Guidelines}

This work adheres to the quality reporting guidelines applicable to protocols of randomized controlled trials. A SPIRIT guideline checklist was submitted with this manuscript documenting compliance.

\section{Authors' Contributions}

LS and $\mathrm{CH}$ led the development of project design and protocols. RM led the development of statistical analysis design and protocols. PP and KR led the development of sleep measurement design and protocols and advised regarding outcomes measurement. RB and EM led the development of corporation 
recruitment protocols and advised regarding outcomes measurement. BF and CB led the development of sleep intervention protocols with advising from KR and LF. MM led the development of qualitative interview protocols. JL led manuscript writing. All authors advised regarding research design and overall protocols. All authors read and approved the final manuscript.

\section{Acknowledgements}

Authors would like to acknowledge the significant support and diligent work of Brian Cox (Project Manager, University of Alabama). Authors also acknowledge the careful work of Teddy Bishop in preparing the manuscript for submission. Lastly, and most importantly, we would like to thank all the nursing home residents and front-line staff participants, and nursing home leaders and corporate partners, past and future, for their dedicated and committed work to improve nursing home resident's lives through pragmatic trials such as this.

\section{References}

1. McCrae CS, McNamara JP, Rowe MA, Dzierzewski JM, Dirk J, Marsiske M, et al. Sleep and affect in older adults: using multilevel modeling to examine daily associations. J Sleep Res. 2008;17(1):42-53.

2. Song Y, Blackwell T, Yaffe K, Ancoli-Israel S, Redline S, Stone KL. Relationships between sleep stages and changes in cognitive function in older men: the MrOS Sleep Study. Sleep. 2015;38(3):411-21.

3. Song Y, Dzierzewski JM, Fung CH, Rodriguez JC, Jouldjian S, Mitchell MN, et al. Association Between Sleep and Physical Function in Older Veterans in an Adult Day Healthcare Program. J Am Geriatr Soc. 2015;63(8):1622-7.

4. Suh SW, Han JW, Lee JR, Byun S, Kwon SJ, Oh SH, et al. Sleep and cognitive decline: A prospective nondemented elderly cohort study. Ann Neurol. 2018;83(3):472-82.

5. da Silva AA, de Mello RG, Schaan CW, Fuchs FD, Redline S, Fuchs SC. Sleep duration and mortality in the elderly: a systematic review with meta-analysis. BMJ Open. 2016;6(2):e008119.

6. Dam TT, Ewing S, Ancoli-Israel S, Ensrud K, Redline S, Stone K. Association between sleep and physical function in older men: the osteoporotic fractures in men sleep study. J Am Geriatr Soc. 2008;56(9):1665-73.

7. Livingston G, Blizard B, Mann A. Does sleep disturbance predict depression in elderly people? A study in inner London. Br J Gen Pract. 1993;43(376):445-8.

8. Miner B, Kryger MH. Sleep in the Aging Population. Sleep Med Clin. 2017;12(1):31-8.

9. St George RJ, Delbaere K, Williams P, Lord SR. Sleep quality and falls in older people living in selfand assisted-care villages. Gerontology. 2009;55(2):162-8.

10. Stenholm S, Head J, Kivimäki M, Magnusson Hanson LL, Pentti J, Rod NH, et al. Sleep Duration and Sleep Disturbances as Predictors of Healthy and Chronic Disease-Free Life Expectancy Between Ages 50 and 75: A Pooled Analysis of Three Cohorts. J Gerontol A Biol Sci Med Sci. 2019;74(2):20410. 
11. Harris-Kojetin L, Sengupta M, Park-Lee E, Valverde R. Long-Term Care Services in the United States: 2013 Overview. Vital Health Stat 3. 2013(37):1-107.

12. Kume Y, Kodama A, Sato K, Kurosawa S, Ishikawa T, Ishikawa S. Sleep/awake status throughout the night and circadian motor activity patterns in older nursing-home residents with or without dementia, and older community-dwelling people without dementia. Int Psychogeriatr. 2016;28(12):2001-8.

13. Wennberg AMV, Wu MN, Rosenberg PB, Spira AP. Sleep Disturbance, Cognitive Decline, and Dementia: A Review. Semin Neurol. 2017;37(4):395-406.

14. Garcia-Alberca JM, Lara JP, Cruz B, Garrido V, Gris E, Barbancho MA. Sleep disturbances in Alzheimer's disease are associated with neuropsychiatric symptoms and antidementia treatment. J Nerv Ment Dis. 2013;201(3):251-7.

15. Koschwanez HE, Kerse N, Darragh M, Jarrett P, Booth RJ, Broadbent E. Expressive writing and wound healing in older adults: a randomized controlled trial. Psychosom Med. 2013;75(6):581-90.

16. Kurdziel LBF, Mantua J, Spencer RMC. Novel word learning in older adults: A role for sleep? Brain Lang. 2017;167:106-13.

17. Valenza MC, Cabrera-Martos I, Martín-Martín L, Pérez-Garzón VM, Velarde C, Valenza-Demet G. Nursing homes: impact of sleep disturbances on functionality. Arch Gerontol Geriatr. 2013;56(3):4326.

18. Ouslander JG, Ai-Samarrai N, Schnelle JF. Prompted voiding for nighttime incontinence in nursing homes: is it effective? J Am Geriatr Soc. 2001;49(6):706-9.

19. Schnelle JF, Alessi CA, Al-Samarrai NR, Fricker RD, Jr., Ouslander JG. The nursing home at night: effects of an intervention on noise, light, and sleep. J Am Geriatr Soc. 1999;47(4):430-8.

20. Schnelle JF, Cruise PA, Alessi CA, Ludlow K, al-Samarrai NR, Ouslander JG. Sleep hygiene in physically dependent nursing home residents: behavioral and environmental intervention implications. Sleep. 1998;21(5):515-23.

21. Schnelle JF, Ouslander JG, Simmons SF, Alessi CA, Gravel MD. The nighttime environment, incontinence care, and sleep disruption in nursing homes. J Am Geriatr Soc. 1993;41(9):910-4.

22. Anderiesen H, Scherder EJ, Goossens RH, Sonneveld MH. A systematic review-physical activity in dementia: the influence of the nursing home environment. Appl Ergon. 2014;45(6):1678-86.

23. Ice GH. Daily life in a nursing home: Has it changed in 25 years? Journal of aging studies. 2002;16(4):345-59.

24. Brimelow RE, Wollin JA, Byrne GJ, Dissanayaka NN. Prescribing of psychotropic drugs and indicators for use in residential aged care and residents with dementia. Int Psychogeriatr. 2019;31(6):837-47.

25. Simpson KM, Richards KC, Enderlin CA, O'Sullivan PS, Koehn M. Medications and sleep in nursing home residents with dementia. Journal of the American Psychiatric Nurses Association. 2006;12(5):279-85.

26. Ruths S, Straand J, Nygaard HA, Bjorvatn B, Pallesen S. Effect of antipsychotic withdrawal on behavior and sleep/wake activity in nursing home residents with dementia: a randomized, placebo- 
controlled, double-blinded study. The Bergen District Nursing Home Study. J Am Geriatr Soc. 2004;52(10):1737-43.

27. Finan PH, Goodin BR, Smith MT. The association of sleep and pain: an update and a path forward. J Pain. 2013;14(12):1539-52.

28. Valenza MC, Cabrera-Martos I, Martin-Martin L, Perez-Garzon VM, Velarde C, Valenza-Demet G. Nursing homes: impact of sleep disturbances on functionality. Arch Gerontol Geriatr. 2013;56(3):4326.

29. Parmelee PA, Tighe CA, Dautovich ND. Sleep disturbance in osteoarthritis: linkages with pain, disability, and depressive symptoms. Arthritis Care Res (Hoboken). 2015;67(3):358-65.

30. Tractenberg RE, Singer CM, Kaye JA. Symptoms of sleep disturbance in persons with Alzheimer's disease and normal elderly. Journal of sleep research. 2005;14(2):177-85.

31. Capezuti E, Sagha Zadeh R, Pain K, Basara A, Jiang NZ, Krieger AC. A systematic review of nonpharmacological interventions to improve nighttime sleep among residents of long-term care settings. BMC Geriatr. 2018;18(1):143.

32. Ye L, Richards KC. Sleep and Long-Term Care. Sleep Med Clin. 2018;13(1):117-25.

33. Pillai JA, Leverenz JB. Sleep and Neurodegeneration: A Critical Appraisal. Chest. 2017;151(6):137586.

34. Ellmers T, Arber S, Luff R, Eyers I, Young E. Factors affecting residents' sleep in care homes. Nurs Older People. 2013;25(8):29-32.

35. Harris $M$, Grando V. When is nighttime? A description of bedtime in persons with dementia in the nursing home. Geriatr Nurs. 2014;35(6):474-8.

36. Arling G, Cooke V, Lewis T, Perkins A, Grabowski DC, Abrahamson K. Minnesota's provider-initiated approach yields care quality gains at participating nursing homes. Health Aff (Millwood). 2013;32(9):1631-8.

37. Collier E, Harrington C. Staffing characteristics, turnover rates, and quality of resident care in nursing facilities. Res Gerontol Nurs. 2008;1(3):157-70.

38. Anderson RA, Issel LM, McDaniel RR, Jr. Nursing homes as complex adaptive systems: relationship between management practice and resident outcomes. Nurs Res. 2003;52(1):12-21.

39. Forbes-Thompson S, Leiker T, Bleich MR. High-performing and low-performing nursing homes: a view from complexity science. Health Care Manage Rev. 2007;32(4):341-51.

40. Low LF, Fletcher J, Goodenough B, Jeon YH, Etherton-Beer C, MacAndrew M, et al. A Systematic Review of Interventions to Change Staff Care Practices in Order to Improve Resident Outcomes in Nursing Homes. PLoS One. 2015;10(11):e0140711.

41. Brady C, Farrell D, Frank B. A Long-term Care Leader's Guide to High Performance: Doing Better Together: Health Professions Press, Incorporated; 2018.

42. Melton L, Lengerich A, Collins M, McKeehan R, Dunn D, Griggs P, et al. Evaluation of Huddles: A Multisite Study. Health Care Manag (Frederick). 2017;36(3):282-7. 
43. Stapley E, Sharples E, Lachman P, Lakhanpaul M, Wolpert M, Deighton J. Factors to consider in the introduction of huddles on clinical wards: perceptions of staff on the SAFE programme. Int J Qual Health Care. 2018;30(1):44-9.

44. Walsh A, Moore A, Everson J, DeCaire K. Gathering, strategizing, motivating and celebrating: the team huddle in a teaching general practice. Educ Prim Care. 2018;29(2):94-9.

45. Brass SD, Olney G, Glimp R, Lemaire A, Kingston M. Using the Patient Safety Huddle as a Tool for High Reliability. Jt Comm J Qual Patient Saf. 2018;44(4):219-26.

46. Newman RE, Bingler MA, Bauer PN, Lee BR, Mann KJ. Rates of ICU Transfers After a Scheduled Night-Shift Interprofessional Huddle. Hosp Pediatr. 2016;6(4):234-42.

47. Tielbur BR, Rice Cella DE, Currie A, Roach JD, Mattingly B, Boone J, et al. Discharge huddle outfitted with mobile technology improves efficiency of transitioning stroke patients into follow-up care. Am J Med Qual. 2015;30(1):36-44.

48. Hartmann CW, Mills WL, Pimentel CB, Palmer JA, Allen RS, Zhao S, et al. Impact of Intervention to Improve Nursing Home Resident-Staff Interactions and Engagement. Gerontologist. 2018;58(4):e291e301.

49. Mills WL, Pimentel CB, Palmer JA, Snow AL, Wewiorski NJ, Allen RS, et al. Applying a Theory-Driven Framework to Guide Quality Improvement Efforts in Nursing Homes: The LOCK Model. Gerontologist. 2018;58(3):598-605.

50. Dewar B, MacBride T. Developing Caring Conversations in care homes: an appreciative inquiry. Health Soc Care Community. 2017;25(4):1375-86.

51. Vogt K, Johnson F, Fraser V, Koh JC, McQueen K, Thornhill J, et al. An Innovative, Strengths-Based, Peer Mentoring Approach to Professional Development for Registered Dietitians. Can J Diet Pract Res. 2015;76(4):185-9.

52. Yanes AF, McElroy LM, Abecassis ZA, Holl J, Woods D, Ladner DP. Observation for assessment of clinician performance: a narrative review. BMJ Qual Saf. 2016;25(1):46-55.

53. Phillips J, Hebish LJ, Mann S, Ching JM, Blackmore CC. Engaging Frontline Leaders and Staff in Real-Time Improvement. Jt Comm J Qual Patient Saf. 2016;42(4):170-83.

54. Dewar B, Barrie K, Sharp C, Meyer J. Implementation of a Complex Intervention to Support Leadership Development in Nursing Homes: A Multimethod Participatory Study. J Appl Gerontol. 2019;38(7):931-58.

55. Sakai M, Naruse T, Nagata S. Relational coordination among home healthcare professions and goal attainment in nursing care. Jpn J Nurs Sci. 2016;13(3):402-10.

56. Pimentel CB, Mills WL, Palmer JA, Dillon K, Sullivan JL, Wewiorski NJ, et al. Blended Facilitation as an Effective Implementation Strategy for Quality Improvement and Research in Nursing Homes. J Nurs Care Qual. 2019;34(3):210-6.

57. Farrell D, Brady C, Frank B. Meeting the leadership challenge in long-term care: What you do matters: Health Professions; 2011. 
58. Smith D. Assessment of the Critical Access Nursing Home Pilot: Final Report. Vineyeard Haven, MA: Advancing Excellence and Drexel University School of Public Health; 2012.

59. Keith RE, Crosson JC, O'Malley AS, Cromp D, Taylor EF. Using the Consolidated Framework for Implementation Research (CFIR) to produce actionable findings: a rapid-cycle evaluation approach to improving implementation. Implement Sci. 2017;12(1):15.

60. Kirk MA, Kelley C, Yankey N, Birken SA, Abadie B, Damschroder L. A systematic review of the use of the Consolidated Framework for Implementation Research. Implement Sci. 2016;11:72.

61. Damschroder LJ, Aron DC, Keith RE, Kirsh SR, Alexander JA, Lowery JC. Fostering implementation of health services research findings into practice: a consolidated framework for advancing implementation science. Implement Sci. 2009;4:50.

62. de Zambotti M, Goldstone A, Claudatos S, Colrain IM, Baker FC. A validation study of Fitbit Charge $2^{\text {TM }}$ compared with polysomnography in adults. Chronobiol Int. 2018;35(4):465-76.

63. Kolla BP, Mansukhani S, Mansukhani MP. Consumer sleep tracking devices: a review of mechanisms, validity and utility. Expert Rev Med Devices. 2016;13(5):497-506.

64. Marino M, Li Y, Rueschman MN, Winkelman JW, Ellenbogen JM, Solet JM, et al. Measuring sleep: accuracy, sensitivity, and specificity of wrist actigraphy compared to polysomnography. Sleep. 2013;36(11):1747-55.

65. Baron KG, Duffecy J, Berendsen MA, Cheung Mason I, Lattie EG, Manalo NC. Feeling validated yet? A scoping review of the use of consumer-targeted wearable and mobile technology to measure and improve sleep. Sleep Med Rev. 2018;40:151-9.

66. Chambers DA, Glasgow RE, Stange KC. The dynamic sustainability framework: addressing the paradox of sustainment amid ongoing change. Implement Sci. 2013;8:117.

67. Tabak RG, Duggan K, Smith C, Aisaka K, Moreland-Russell S, Brownson RC. Assessing Capacity for Sustainability of Effective Programs and Policies in Local Health Departments. J Public Health Manag Pract. 2016;22(2):129-37.

68. Vitale R, Blaine T, Zofkie E, Moreland-Russell S, Combs T, Brownson RC, et al. Developing an evidence-based program sustainability training curriculum: a group randomized, multi-phase approach. Implement Sci. 2018;13(1):126.

69. Centers for Medicare and Medicaid. Nursing home data compendium 2015 edition. Washington, DC. 2015. https://www.cms.gov/Medicare/Provider-Enrollment-andCertification/CertificationandComplianc/Downloads/nursinghomedatacompendium_508-2015.pdf. Accessed December 14, 2019.

70. National Center for Health Statistics. Alzheimer's Disease 2016. Available from: https://www.cdc.gov/nchs/fastats/alzheimers.htm. Accessed January 15, 2019.

71. Woertman W, de Hoop E, Moerbeek M, Zuidema SU, Gerritsen DL, Teerenstra S. Stepped wedge designs could reduce the required sample size in cluster randomized trials. J Clin Epidemiol. 2013;66(7):752-8. 
72. Chung F, Subramanyam R, Liao P, Sasaki E, Shapiro C, Sun Y. High STOP-Bang score indicates a high probability of obstructive sleep apnoea. Br J Anaesth. 2012;108(5):768-75.

73. Chung F, Yang Y, Brown R, Liao P. Alternative scoring models of STOP-bang questionnaire improve specificity to detect undiagnosed obstructive sleep apnea. J Clin Sleep Med. 2014;10(9):951-8.

74. Chung F, Yegneswaran B, Liao P, Chung SA, Vairavanathan S, Islam S, et al. STOP questionnaire: a tool to screen patients for obstructive sleep apnea. Anesthesiology. 2008;108(5):812-21.

75. Smith MT, McCrae CS, Cheung J, Martin JL, Harrod CG, Heald JL, et al. Use of Actigraphy for the Evaluation of Sleep Disorders and Circadian Rhythm Sleep-Wake Disorders: An American Academy of Sleep Medicine Systematic Review, Meta-Analysis, and GRADE Assessment. J Clin Sleep Med. 2018;14(7):1209-30.

76. Maglione JE, Liu L, Neikrug AB, Poon T, Natarajan L, Calderon J, et al. Actigraphy for the assessment of sleep measures in Parkinson's disease. Sleep. 2013;36(8):1209-17.

77. Boland J, Currow DC, Wilcock A, Tieman J, Hussain JA, Pitsillides C, et al. A systematic review of strategies used to increase recruitment of people with cancer or organ failure into clinical trials: implications for palliative care research. J Pain Symptom Manage. 2015;49(4):762-72.e5.

78. Hunt KJ, Shlomo N, Addington-Hall J. Participant recruitment in sensitive surveys: a comparative trial of 'opt in' versus 'opt out' approaches. BMC Med Res Methodol. 2013;13:3.

79. Miller CJ, Burgess JF, Jr., Fischer EP, Hodges DJ, Belanger LK, Lipschitz JM, et al. Practical application of opt-out recruitment methods in two health services research studies. BMC Med Res Methodol. 2017;17(1):57.

80. Vellinga A, Cormican M, Hanahoe B, Bennett K, Murphy AW. Opt-out as an acceptable method of obtaining consent in medical research: a short report. BMC Med Res Methodol. 2011;11:40.

81. Chodosh J, Edelen MO, Buchanan JL, Yosef JA, Ouslander JG, Berlowitz DR, et al. Nursing home assessment of cognitive impairment: development and testing of a brief instrument of mental status. J Am Geriatr Soc. 2008;56(11):2069-75.

82. Hemming K, Lilford R, Girling AJ. Stepped-wedge cluster randomised controlled trials: a generic framework including parallel and multiple-level designs. Stat Med. 2015;34(2):181-96.

83. Bellone GJ, Plano SA, Cardinali DP, Chada DP, Vigo DE, Golombek DA. Comparative analysis of actigraphy performance in healthy young subjects. Sleep Sci. 2016;9(4):272-9.

84. Buysse DJ, Hall ML, Strollo PJ, Kamarck TW, Owens J, Lee L, et al. Relationships between the Pittsburgh Sleep Quality Index (PSQI), Epworth Sleepiness Scale (ESS), and clinical/polysomnographic measures in a community sample. J Clin Sleep Med. 2008;4(6):563-71.

85. Zhu B, Bronas UG, Fritschi C. Sleep assessment in aging adults with type 2 diabetes: agreement between actigraphy and sleep diaries. Sleep Med. 2018;46:88-94.

86. Berk M, Ng F, Dodd S, Callaly T, Campbell S, Bernardo M, et al. The validity of the CGI severity and improvement scales as measures of clinical effectiveness suitable for routine clinical use. J Eval Clin Pract. 2008;14(6):979-83. 
87. Black JE, Hull SG, Tiller J, Yang R, Harsh JR. The long-term tolerability and efficacy of armodafinil in patients with excessive sleepiness associated with treated obstructive sleep apnea, shift work disorder, or narcolepsy: an open-label extension study. J Clin Sleep Med. 2010;6(5):458-66.

88. Busner J, Targum SD. The clinical global impressions scale: applying a research tool in clinical practice. Psychiatry (Edgmont). 2007;4(7):28-37.

89. Dunlop BW, Gray J, Rapaport MH. Transdiagnostic Clinical Global Impression Scoring for Routine Clinical Settings. Behav Sci (Basel). 2017;7(3).

90. Dworkin RH, Turk DC, Farrar JT, Haythornthwaite JA, Jensen MP, Katz NP, et al. Core outcome measures for chronic pain clinical trials: IMMPACT recommendations. Pain. 2005;113(1-2):9-19.

91. Farrar JT, Young JP, Jr., LaMoreaux L, Werth JL, Poole RM. Clinical importance of changes in chronic pain intensity measured on an 11-point numerical pain rating scale. Pain. 2001;94(2):149-58.

92. Schneider LS, Olin JT, Doody RS, Clark CM, Morris JC, Reisberg B, et al. Validity and reliability of the Alzheimer's Disease Cooperative Study-Clinical Global Impression of Change. The Alzheimer's Disease Cooperative Study. Alzheimer Dis Assoc Disord. 1997;11 Suppl 2:S22-32.

93. Teri L, Logsdon RG, Peskind E, Raskind M, Weiner MF, Tractenberg RE, et al. Treatment of agitation in AD: a randomized, placebo-controlled clinical trial. Neurology. 2000;55(9):1271-8.

94. Saliba D, Buchanan J. Making the investment count: revision of the Minimum Data Set for nursing homes, MDS 3.0. J Am Med Dir Assoc. 2012;13(7):602-10.

95. Saliba D, Buchanan J. Development \& Validation of a Revised Nursing Home Assessment Tool: MDS 3.0. 2008. Santa Monica, CA: RAND Health Coorporation. 2009.

96. Bellows NM, Halpin HA. MDS-based state Medicaid reimbursement and the ADL-decline quality indicator. Gerontologist. 2008;48(3):324-9.

97. Estabrooks CA, Knopp-Sihota JA, Norton PG. Practice sensitive quality indicators in RAI-MDS 2.0 nursing home data. BMC Res Notes. 2013;6:460.

98. Saliba D, Solomon D, Rubenstein L, Young R, Schnelle J, Roth C, et al. Quality indicators for the management of medical conditions in nursing home residents. J Am Med Dir Assoc. 2005;6(3 Suppl):S36-48.

99. RTI International. MDS 3.0 Quality Measures Users Manual. Washington, DC: Centers for Medicare and Medicaid Services; 2016.

100. Richards K, Shue VM, Beck CK, Lambert CW, Bliwise DL. Restless legs syndrome risk factors, behaviors, and diagnoses in persons with early to moderate dementia and sleep disturbance. Behav Sleep Med. 2010;8(1):48-61.

101. Richards KC, Lambert C, Beck CK, Bliwise DL, Evans WJ, Kalra GK, et al. Strength training, walking, and social activity improve sleep in nursing home and assisted living residents: randomized controlled trial. J Am Geriatr Soc. 2011;59(2):214-23.

102. Rose KM, Beck C, Tsai PF, Liem PH, Davila DG, Kleban M, et al. Sleep disturbances and nocturnal agitation behaviors in older adults with dementia. Sleep. 2011;34(6):779-86. 
103. King N. Using templates in the thematic analysis of text. Essential Guide to Qualitative Methods in Organizational Research. London: SAGE Publications Ltd; 2004.

104. Beebe J. Basic concepts and techniques of rapid appraisal. Human organization. 1995;54(1):42-51.

105. Hussey MA, Hughes JP. Design and analysis of stepped wedge cluster randomized trials. Contemp Clin Trials. 2007;28(2):182-91.

106. Gittell JH, Godfrey M, Thistlethwaite J. Interprofessional collaborative practice and relational coordination: improving healthcare through relationships. J Interprof Care. 2013;27(3):210-3.

107. Weinberg DB, Lusenhop RW, Gittell JH, Kautz CM. Coordination between formal providers and informal caregivers. Health Care Manage Rev. 2007;32(2):140-9.

108. O'Neill C, Harrington C, Kitchener M, Saliba D. Quality of care in nursing homes: an analysis of relationships among profit, quality, and ownership. Med Care. 2003;41(12):1318-30.

109. Trinkoff AM, Storr CL, Lerner NB, Yang BK, Han K. CNA Training Requirements and Resident Care Outcomes in Nursing Homes. Gerontologist. 2017;57(3):501-8.

110. Marshall A, Altman DG, Holder RL, Royston P. Combining estimates of interest in prognostic modelling studies after multiple imputation: current practice and guidelines. BMC Med Res Methodol. 2009;9:57.

111. Keith RE, Hopp FP, Subramanian U, Wiitala W, Lowery JC. Fidelity of implementation: development and testing of a measure. Implement Sci. 2010;5:99.

112. Bernard HR. Research methods in anthropology: Qualitative and quantitative approaches: Rowman \& Littlefield; 2017.

113. Patton MQ. Qualitative research \& evaluation methods: Integrating theory and practice: Sage publications; 2014.

114. Miles MB, Huberman AM, Saldaña J. Qualitative data analysis: A methods sourcebook. 3rd. Thousand Oaks, CA: Sage; 2014.

115. Castle NG, Engberg J, Anderson R, Men A. Job satisfaction of nurse aides in nursing homes: intent to leave and turnover. Gerontologist. 2007;47(2):193-204.

116. Office of the Assistant Secretary for Planning and Evaluation. National Plan to Address Alzheimer's Disease: 2018 Update. Washington, DC: US Department of Health and Human Services; 2018. https://aspe.hhs.gov/system/files/pdf/259581/NatPlan2018.pdf. Accessed Jan 21, 2019.

\section{Tables}

Table 1: Stepped Wedge Design with Measurement Periods 


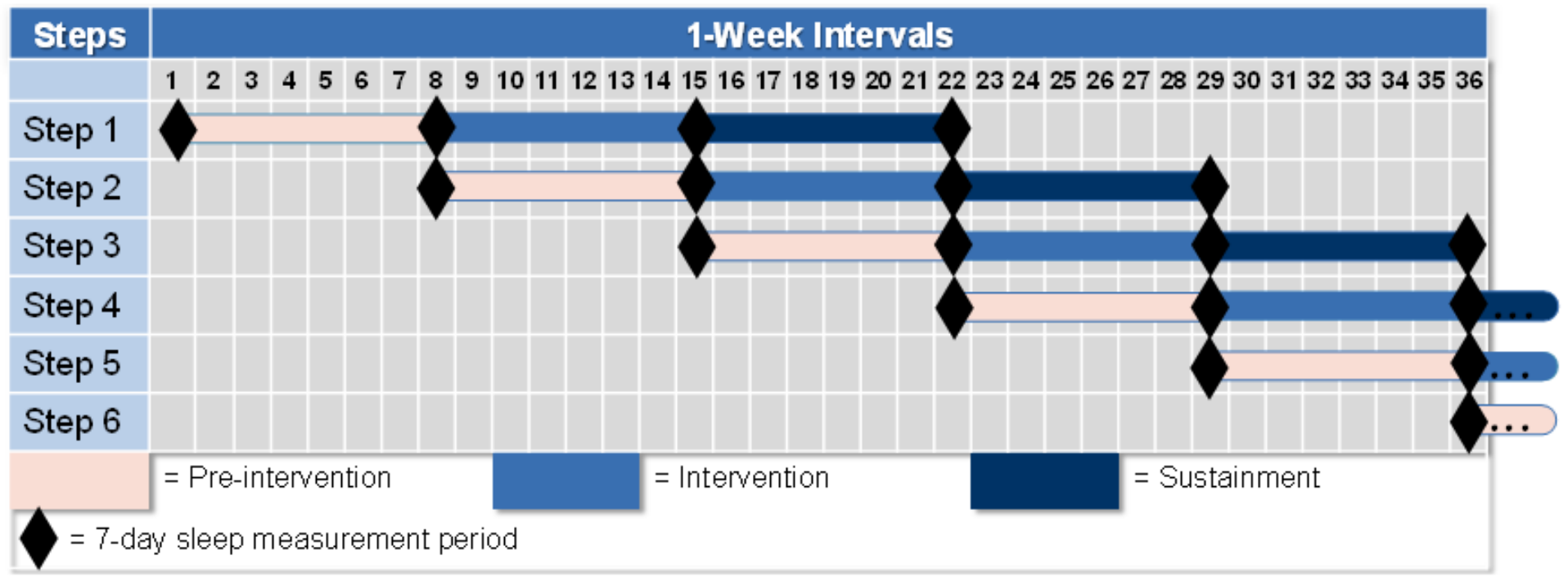

Table 2 Primary, Secondary, and Other Outcome Measures 


\begin{tabular}{|c|c|c|c|}
\hline Category & Name & Time Frame & Brief Description \\
\hline Primary & $\begin{array}{l}\text { Total Sleep } \\
\text { Time via } \\
\text { Actigraph }\end{array}$ & $\begin{array}{l}\text { Baseline, } \\
\text { Intervention, } \\
\text { Post- } \\
\text { Treatment }\end{array}$ & $\begin{array}{l}\text { Actigraph measurement of the total number of minutes } \\
\text { the subject is asleep between } 7 \mathrm{pm} \\
\text { and 7am. }\end{array}$ \\
\hline Secondary & $\begin{array}{l}\text { Psychotropic } \\
\text { Medication } \\
\text { Use }\end{array}$ & $\begin{array}{l}\text { Baseline, } \\
\text { Intervention, } \\
\text { Post- } \\
\text { Treatment }\end{array}$ & $\begin{array}{l}\text { Psychotropic medication use as recorded in the } \\
\text { Minimum Data Set (MDS) }\end{array}$ \\
\hline Secondary & $\begin{array}{l}\text { Pain and } \\
\text { Analgesic } \\
\text { Medication } \\
\text { Use }\end{array}$ & $\begin{array}{l}\text { Baseline, } \\
\text { Intervention, } \\
\text { Post- } \\
\text { Treatment }\end{array}$ & $\begin{array}{l}\text { Psychotropic medication use as recorded in the } \\
\text { Minimum Data Set (MDS) }\end{array}$ \\
\hline Secondary & $\begin{array}{l}\text { Activities of } \\
\text { Daily Living } \\
\text { Decline }\end{array}$ & $\begin{array}{l}\text { Baseline, } \\
\text { Intervention, } \\
\text { Post- } \\
\text { Treatment }\end{array}$ & $\begin{array}{l}\text { Activities of daily living as recorded in the Minimum } \\
\text { Data Set (MDS) }\end{array}$ \\
\hline Other & $\begin{array}{l}\text { Sleep Staff } \\
\text { Rating }\end{array}$ & $\begin{array}{l}\text { Intervention, } \\
\text { Post- } \\
\text { Treatment }\end{array}$ & Staff rating of sleep global impression of change \\
\hline Other & $\begin{array}{l}\text { Staff- } \\
\text { identified } \\
\text { Sleep-related } \\
\text { Concerns }\end{array}$ & $\begin{array}{l}\text { Intervention, } \\
\text { Post- } \\
\text { Treatment }\end{array}$ & $\begin{array}{l}\text { Staff rating of sleep-related concerns global impression } \\
\text { of change }\end{array}$ \\
\hline Other & $\begin{array}{l}\text { Inter-resident } \\
\text { Sleep } \\
\text { Variability }\end{array}$ & $\begin{array}{l}\text { Baseline, } \\
\text { Intervention, } \\
\text { Post- } \\
\text { Treatment }\end{array}$ & $\begin{array}{l}\mathrm{NH} \text { medical record data to indicate (a) changes in any } \\
\text { sedating medications and } \\
\text { changes in dosages; ( } 2 \text { ) incidents of delirium; ( } 3 \text { ) any } \\
\text { urinary tract infections; (4) doses of any sedating } \\
\text { medications, including as } \\
\text { needed ones }\end{array}$ \\
\hline Other & $\begin{array}{l}\text { Sleep } \\
\text { Information } \\
\text { from MDS }\end{array}$ & $\begin{array}{l}\text { Baseline, } \\
\text { Intervention, } \\
\text { Post- } \\
\text { Treatment }\end{array}$ & $\begin{array}{l}\text { The MDS contains only two items pertaining to sleep. } \\
\text { Because MDS is the foundational nursing home } \\
\text { administrative quality data set, } \\
\text { these items will be examined for their utility. One item, } \\
\text { part of the PHQ-9, is, "trouble falling or staying asleep } \\
\text { or sleeping too much." The pain section also includes } \\
\text { one item for residents who can self-report, "over the } \\
\text { past } 5 \text { days, has pain made it hard for } \\
\text { you to sleep at night" (there is not a comparable item in } \\
\text { the MDS staff interview section for residents unable to } \\
\text { Page } 27 / 31\end{array}$ \\
\hline
\end{tabular}


self-report).

\section{Table 3: CFIR Implementation Constructs and Data Sources}

\begin{tabular}{|c|c|}
\hline CFIR Domains \& Constructs & Assessments \\
\hline Intervention Characteristics & Staff Interviews \\
\hline $\begin{array}{l}\text { Relative Advantage [of } \\
\text { intervention] }\end{array}$ & Frequency of actigraph use per resident \\
\hline Process & - $\quad$ Staff interviews \\
\hline - $\quad$ Planning & Number of actigraphs used with residents \\
\hline - $\quad$ Engaging & Amount of supplementary sleep data sent to researchers \\
\hline - $\quad$ Executing & \\
\hline $\begin{array}{l}\text { Reflecting and } \\
\text { Evaluating }\end{array}$ & \\
\hline Inner setting [of NHs] & - $\quad$ Staff interviews \\
\hline $\begin{array}{l}\quad \text { Structural } \\
\text { characteristics }\end{array}$ & $\begin{array}{l}\text { - NH characteristics (payment mix, staffing size, chain and } \\
\text { ownership status) from CASPER }\end{array}$ \\
\hline Networks and & Staff attendance at trainings and coaching calls \\
\hline 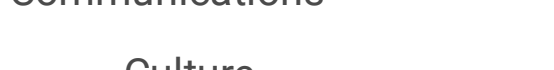 & - $\quad$ Leadership team turnover during intervention \\
\hline - Implementation & - $\quad$ Frequency of STOP-Bang use \\
\hline Outer Setting & - $\quad$ Staff interviews \\
\hline $\begin{array}{l}\text { Peer Pressure [to } \\
\text { implement] }\end{array}$ & $\begin{array}{l}\text { Number of non-trial NHs within a corporation implementing } \\
\text { the LOCK sleep intervention }\end{array}$ \\
\hline
\end{tabular}

\section{Figures}




\begin{tabular}{|c|c|c|}
\hline $\begin{array}{l}\text { LOCK } \\
\text { Element }\end{array}$ & $\begin{array}{c}\text { Evidence-Based Concept \& } \\
\text { Explanation }\end{array}$ & Fictional Example Relating to Sleep \\
\hline $\begin{array}{l}\text { Learn from } \\
\text { bright spots }\end{array}$ & $\begin{array}{l}\text { Strengths-based learning: } \\
\text { When searching for solutions } \\
\text { to an issue, look for positive } \\
\text { outliers (i.e., positive deviants) } \\
\text { to identify instances of success } \\
\text { from which to learn. }\end{array}$ & $\begin{array}{l}\text { Ms. Smith doesn't sleep well. But last weekend she slept } \\
\text { straight through two nights in a row. What happened during } \\
\text { that time? Staff investigate and discover Ms. Smith's } \\
\text { roommate's bed alarm broke and wasn't replaced until after } \\
\text { the weekend. And Ms. Smith's daughter was in town and took } \\
\text { her for long walks outside. }\end{array}$ \\
\hline Observe & $\begin{array}{l}\text { Observation: Have staff briefly } \\
\text { step back from their regular } \\
\text { routines and conduct specific } \\
\text { observations to collect data, } \\
\text { using structured tools or not. } \\
\text { These observations provide the } \\
\text { fodder for huddle dialogues. }\end{array}$ & $\begin{array}{l}\text { A main contributor to Ms. Smith's poor sleep is her low } \\
\text { daytime activity level and noise at night. Nursing uses an } \\
\text { actigraph to observe the quantity and quality of her sleep at } \\
\text { night. Nursing and recreation work together to observe and } \\
\text { informally track Ms. Smith's daytime activity level. All staff in } \\
\text { the neighborhood observe indications of Ms. Smith's activity } \\
\text { and behavior during the day and night, particularly in } \\
\text { response to noise. }\end{array}$ \\
\hline $\begin{array}{l}\text { Collaborate } \\
\text { in huddles }\end{array}$ & $\begin{array}{l}\text { Relationship-based teams: } \\
\text { Conduct brief, collaborative, } \\
\text { strengths-based frontline staff } \\
\text { huddles to discuss risk factors } \\
\text { for an issue, bright spots, } \\
\text { results of observations, and } \\
\text { action planning. }\end{array}$ & $\begin{array}{l}\text { In a frontline staff huddle, the facilitator moves the } \\
\text { conversation about Ms. Smith through the steps of bright spot } \\
\text { exploration, hypothesis generation based on observation, and } \\
\text { action planning, making sure everyone is heard. Staff decide } \\
\text { to offer Ms. Smith a variety of activities to increase her } \\
\text { daytime activity level. On Monday, the group determines to } \\
\text { pilot } 3 \text { new options. The group agrees to huddle Thursday to } \\
\text { discuss what worked and what other activity options to pilot. }\end{array}$ \\
\hline $\begin{array}{l}\text { Keep it bite } \\
\text { sized }\end{array}$ & $\begin{array}{l}\text { Efficiency: Keep all LOCK } \\
\text { components to } 5-15 \text { minutes. } \\
\text { Incremental changes, rather } \\
\text { than systemic overhauls, are } \\
\text { easier to integrate. }\end{array}$ & $\begin{array}{l}\text { Staff shorten existing meetings by 5-10 minutes to make } \\
\text { room for frontline staff huddles. Staff huddle more frequently } \\
\text { for shorter intervals to facilitate timely information exchange. } \\
\text { Huddle facilitators ensure pilot changes resulting from action } \\
\text { plans are small enough to fit into routines but meaningful. }\end{array}$ \\
\hline
\end{tabular}

\section{Figure 1}

LOCK Elements, Corresponding Evidence-Based Concepts, and Examples 


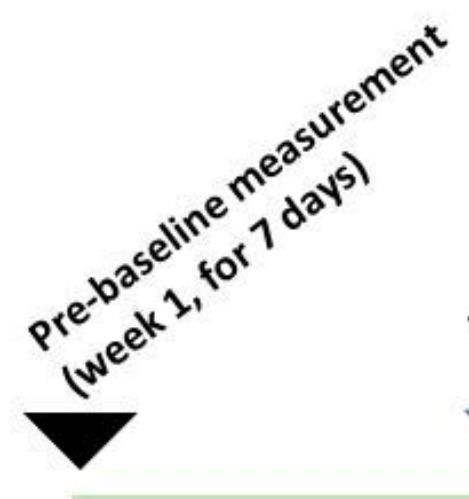

Preintervention (8 weeks)

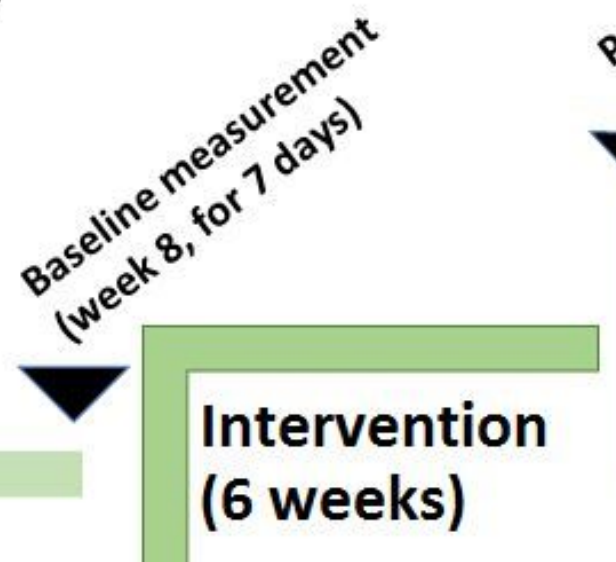

Intervent
(6 weeks)

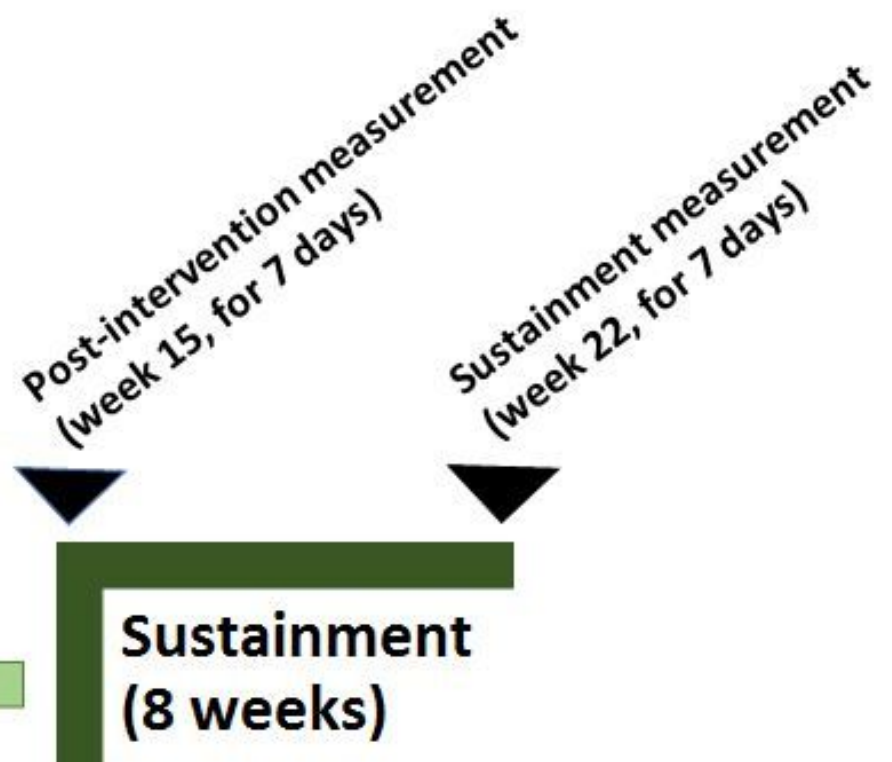

(8 weeks)

\section{Phase Duration: 22 Weeks}

\section{Figure 2}

Timing and Spacing of Intervention Implementation and Sleep Outcomes Measures 


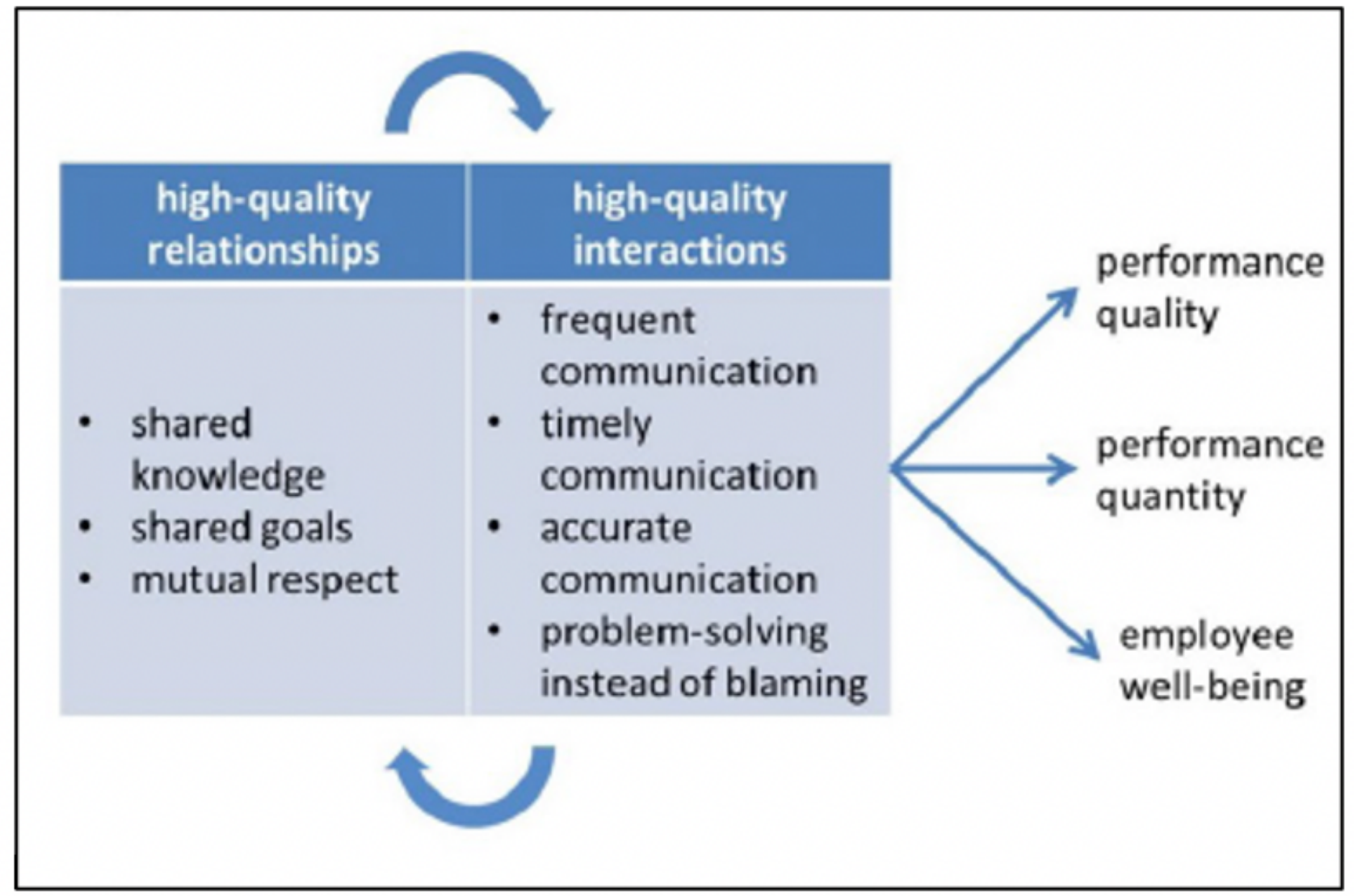

Figure 3

Relational Coordination Theory

\section{Supplementary Files}

This is a list of supplementary files associated with this preprint. Click to download.

- AdditionalFile1.pdf

- AdditionalFile2.docx

- AdditionalFile3.doc 